\title{
(6) OPEN ACCESS \\ A decoy receptor 3 analogue reduces localised defects in phagocyte function in pneumococcal pneumonia
}

ORIGINAL ARTICLE

\author{
Helen M Marriott, ${ }^{1}$ Marc Daigneault, ${ }^{1}$ Alfred A R Thompson, ${ }^{1,2}$ Sarah R Walmsley, ${ }^{1,2}$ \\ Sharonjit K Gill, ${ }^{1}$ Derrick R Witcher, ${ }^{3}$ Victor J Wroblewski, ${ }^{4}$ Paul G Hellewell, ${ }^{5}$ \\ Moira K B Whyte, ${ }^{1,2}$ David H Dockrell ${ }^{1,2}$
}

\begin{abstract}
- Additional materials are published online only. To view these files please visit the journal online (http://dx.doi.org/ 10.1136/

thoraxjnl-2012-201591)

${ }^{1}$ Department of Infection and Immunity, University of Sheffield, Sheffield, UK

${ }^{2}$ Sheffield Teaching Hospitals, Sheffield, UK

${ }^{3}$ Biotechnology Discovery Research, Lilly Research Laboratories, Eli Lilly \& Company, Lilly Corporate Center, Indianapolis, Indiana, USA

${ }^{4}$ Department of Drug Disposition Development/

Commercialization, Lilly

Research Laboratories, Eli Lilly \& Company, Lilly Corporate Center, Indianapolis, Indiana, USA

${ }^{5}$ Department of Cardiovascular Science, University of Sheffield, Sheffield, UK
\end{abstract}

Correspondence to Dr Helen Marriott, Department of Infection and Immunity, University of Sheffield Medical School, Beech Hill Road, Sheffield S10 2RX, UK; h.m. marriott@sheffield.ac.uk

Received 5 January 2012 Accepted 29 May 2012 Published Online First 26 June 2012

\section{ABSTRACT \\ Background Therapeutic strategies to modulate the host response to bacterial pneumonia are needed to improve outcomes during community-acquired pneumonia. This study used mice with impaired Fas signalling to examine susceptibility to pneumococcal pneumonia and decoy receptor 3 analogue (DcR3-a) to correct factors associated with increased susceptibility.}

Methods Wild-type mice and those with varying degrees of impairment of Fas (/pr) or Fas ligand signalling (g/d) were challenged with Streptococcus pneumoniae and microbiological and immunological outcomes measured in the presence or absence of DcR3-a. Results During established pneumonia, neutrophils became the predominant cell in the airway and gld mice were less able to clear bacteria from the lungs, demonstrating localised impairment of pulmonary neutrophil function in comparison to Ipr or wild-type mice. T-cells from gld mice had enhanced activation and reduced apoptosis in comparison to wild-type and $/ p r$ mice during established pneumonia. Treatment with DcR3-a reduced T-cell activation and corrected the defect in pulmonary bacterial clearance in gld mice. Conclusions The results suggest that imbalance in tumour necrosis factor superfamily signalling and excessive T-cell activation can impair bacterial clearance in the lung but that DcR3-a treatment can reduce T-cell activation, restore optimal pulmonary neutrophil function and enhance bacterial clearance during $S$ pneumoniae infection.

\section{INTRODUCTION}

Streptococcus pneumoniae (the pneumococcus) is the commonest cause of community-acquired pneumonia (CAP) and causes approximately 2 million deaths annually. ${ }^{1}$ Despite advances in antimicrobial therapy and management of critically ill patients, therapeutic modulation of the pulmonary inflammatory response has been relatively neglected in CAP, even though dysregulation of the inflammatory response is a key feature of pathogenesis. To adopt this strategy we need a better understanding of factors which regulate susceptibility to CAP, and how these can be manipulated to ensure an optimal inflammatory response that achieves bacterial clearance without inflammatory lung injury.

The tumour necrosis factor superfamily (TNFSF) regulates inflammatory responses. In this study we have addressed whether TNFSF member Fas ligand

\section{Key messages}

What is the key question?

- Does tumour necrosis factor (TNF) superfamily member signalling modify outcomes of pneumococcal pneumonia?

What is the bottom line?

- The balance of Fas ligand and decoy receptor 3 inhibitable ligands influences T-cell activation and local neutrophil function in the lung during pneumococcal pneumonia.

\section{Why read on?}

- Immunomodulation of TNF superfamily signalling has potential as immunotherapy during community-acquired pneumonia.

(FasL, TNFSF6) influences susceptibility to pneumococcal pneumonia. FasL is a transmembrane protein that signals through Fas (CD95, TNFRSF6). ${ }^{2}$ Multimerised Fas activates apoptosis in a range of cells, including epithelial cells and phagocytes, regulating immune homeostasis. ${ }^{3}$ Alternatively Fas signal transduction contributes to cell activation and pro-inflammatory effects, for example in the lung FasL contributes to the pathogenesis of acute lung injury. ${ }^{4}$ FasL expression increases during pneumococcal infection. ${ }^{56}$ During pneumonia with Gram-positive bacteria there is less neutrophil recruitment in $1 p r$ mice, which have low levels of functional Fas, or in mice treated with a decoy receptor 3 analogue (DcR3-a) that neutralises FasL. ${ }^{67}$ Despite this, bacterial clearance is enhanced unless a more severe infection is established, suggesting under certain circumstances FasL has an adverse effect on disease outcome. ${ }^{6}$ FasL therefore has key roles in immune homeostasis, $^{8}$ and host defence. ${ }^{9}$ It also has the potential to exacerbate inflammatory lung injury, and its role in bacterial clearance varies depending on the infection and murine model studied. ${ }^{67}$

We addressed the role of FasL in the pathogenesis of CAP in murine models of pneumococcal pneumonia (see online supplement). We tested the role of complete FasL deficiency using C57BL/6 gld mice, which express FasL lacking functional 
activity. ${ }^{10}$ The results were compared with wild-type C57BL/6 mice and C57BL/ 6 mice carrying the lpr mutation, which results in reduced expression of Fas, ${ }^{11}$ but less than complete inhibition of Fas signalling, since the mutation is leaky. ${ }^{12}{ }^{13}$ We also compared results in these mice with the effect of FasL neutralisation with DcR3-a. DcR3-a is an analogue of DcR3 (TNFRSF6B) which neutralises FasL but also inhibits two other TNFSF members: TNFSF14 (lymphotoxin-related inducible ligand which competes for glycoprotein $\mathrm{D}$ binding to herpes virus entry mediator on T cells; LIGHT) and TNFSF15 (TNF-like protein 1A; TL1A). ${ }^{14-16}$ This allowed us to compare the effects of selective versus non-selective FasL neutralisation that also included other TNFSF members to determine their potential role in the pathogenesis of CAP and to examine the therapeutic potential of manipulation of TNFSF signalling during CAP.

Our murine models suggest inhibition of FasL signalling enhances T-cell activation and impedes bacterial clearance but that these alterations can be corrected by administration of a TNFSF decoy receptor 3 analogue (DcR3-a).

\section{MATERIAL AND METHODS Animals}

Adult female mice, homozygous for the FasL ${ }^{\text {gld }}$ mutation (B6Smn. $\left.\mathrm{C}_{3}-\mathrm{Fasl}^{\text {gld }} / \mathrm{J}\right)$ and $\mathrm{Fas}^{\mathrm{lpr}}\left(\mathrm{B} 6 . \mathrm{MRL}-\mathrm{Fas}^{\mathrm{lpr}} / \mathrm{J}\right)$, on a C57BL/6 background (Jackson Laboratories, Bar Harbour, Maine, USA) were used. ${ }^{10}{ }^{11} \mathrm{C} 57 \mathrm{BL} / 6$ mice were used as wild-type controls. All animal experiments were conducted in accordance with the Home Office Animals (Scientific Procedures) Act 1986 and approved by the University of Sheffield Ethical Review Committee.

\section{Pneumococcal infection model}

Pulmonary infection of mice was by direct tracheal instillation as described previously. ${ }^{17}$ Neutrophil depletion was with $100 \mu \mathrm{g} /$ mouse Gr-1 antibody or isotype control. Decoy receptor treatment was with $400 \mu \mathrm{g} /$ mouse DcR3-a or bovine serum albumin (BSA). For detailed methods see online supplement. Viable bacteria in lung and blood were measured and cell differential was assessed by morphology on cytospins. ${ }^{17}$ Apoptosis detection was by nuclear morphology or Annexin V-TopPro 3 staining and flow cytometry, with neutrophils being identified by Ly6G staining and forward/side scatter characteristics as described. ${ }^{17}$

\section{In vitro neutrophil function}

Peripheral blood neutrophils were isolated by negative magnetic selection with cell purity routinely greater than $80 \% .{ }^{18}$ Rates of apoptosis were assessed by morphology on cytospins after $9 \mathrm{~h}$ of culture. For apoptosis after bacterial infection neutrophils were infected with serotype $2 S$ pneumoniae (multiplicity of infection $(\mathrm{MOI})=10)$ and apoptosis assessed at $9 \mathrm{~h}$ post infection by nuclear morphology. Respiratory burst was assessed by flow cytometry analysis of dichlorodihydrofluorescein fluorescence following $30 \mathrm{~min}$ of stimulation by heat-killed serotype $2 \mathrm{~S}$ pneumoniae $(\mathrm{MOI}=10)$. Phagocytosis was assessed by calculating the phagocytic index on cytospins of neutrophils incubated with opsonized zymosan $(20 \mu \mathrm{g} / \mathrm{ml})$ for $30 \mathrm{~min}$; internalised particles were counted as those within a phagocytic vacuole.

\section{Isolation and staining of splenocytes}

Mice received $1 \times 10^{7}$ colony-forming units (CFUs) of serotype $4 \mathrm{~S}$ pneumoniae and after $24 \mathrm{~h}$ were killed by overdose of anaesthetic and exsanguination. Spleens were collected, splenocytes isolated and activation of $\mathrm{CD}^{+}$T cells measured by flow cytometry (LSR II, BD Biosciences, Oxford, UK) as percentage of $\mathrm{CD}^{2} 5^{+}$cells.
Apoptosis was measured as the percentage of cells with hypodiploid DNA after propidium iodide staining. ${ }^{19}$ Analysis was with FlowJo software V.9.3.2 (Tree Star, Inc, Ashland, Oregon, USA).

\section{Statistics}

Statistical analysis was by analysis of variance (parametric data), t test (parametric data), Kruskal-Wallis (non-parametric data), Mann-Whitney (non-parametric data) or log-rank test for survival, using Prism 5.0 software (GraphPad Inc, La Jolla, California, USA). Differences were considered significant if $\mathrm{p}<0.05$.

\section{RESULTS \\ Reduced bacterial clearance in g/d mice with established pneumonia}

We examined the outcome of infection using three models of infection with high inocula of $S$ pneumoniae. There was an increase in CFUs in lung and blood, $24 \mathrm{~h}$ after infection, in the gld mice compared with C57BL/6 and Ipr mice (which had similar CFUs), after both serotype 1 (figure 1A,B) and serotype 2 infection (figure 1D,E). Serotype 4 infections resulted in higher bacterial CFUs in lung or blood of wild-type and lpr mice but the increase in CFUs for gld mice was not significant (figure $1 \mathrm{G}, \mathrm{H}$ ).

FasL contributes to CXC chemokine expression in the lung and to neutrophil recruitment into the airspaces in response to a variety of stimuli including pneumococci. ${ }^{6}{ }^{20}$ We documented impaired neutrophil recruitment into the lung in the absence of Fas signalling in both gld and $l p r$ mice $24 \mathrm{~h}$ after challenge with serotype $1 S$ pneumoniae (figure 1C). After infection with the more virulent serotypes 2 or $4 S$ pneumoniae, neutrophil numbers were similar between strains at $24 \mathrm{~h}$ (figure 1F,I). Fas can trigger neutrophil apoptosis. ${ }^{3}$ Since absolute levels of neutrophil apoptosis in vivo in the lung are influenced by the rate of apoptosis induction and the rate of clearance of apoptotic cells, while the percentage of apoptotic cells is confounded by rates of neutrophil recruitment, we measured rates of constitutive apoptosis in vitro. We confirmed reduced rates of constitutive apoptosis in peripheral blood neutrophils from $l p r$ and gld mice, but found no reduction in neutrophil apoptosis in the presence of pneumococci in vitro and confirmed this finding in vivo (figure $2 \mathrm{~A}-\mathrm{C}$ ).

\section{Dysfunctional pulmonary neutrophil responses in gld mice}

We next addressed the functional capacity of phagocytes. As gld mice had no reduction in alveolar macrophage-dependent bacterial clearance (online supplementary figure 3) we addressed the functional capacity of neutrophils. Since the number of bacteria inside neutrophils reflects rates of ingestion and killing we measured rates of zymosan uptake as a marker of phagocytic capacity. Peripheral blood neutrophils from gld and lpr mice had no intrinsic defect in phagocytosis or in generation of reactive oxygen species when challenged with heat-killed $S$ pneumoniae ex vivo (figure $3 \mathrm{~A}, \mathrm{~B}$ ). To address the contribution of neutrophils to bacterial clearance in vivo we reduced neutrophil numbers with anti-Gr1 antibody (figure 4A, online supplementary figure 2) and examined clearance of serotype $2 S$ pneumoniae at $14 \mathrm{~h}$ after infection, to ensure the phagocytes were recently recruited. ${ }^{21}$ Although anti-Gr-1 also depletes monocytes these made up only about $1 \%$ of bronchial alveolar lavage cells at this time point. The anti-Gr-1 treatment significantly increased CFUs in the lung and blood of C57BL/ 6 and lpr mice but not gld mice (figure 4B,C). Since the defect in bacterial CFUs was more marked for gld mice in blood than in lung we also investigated whether these mice were less able to clear bacteria from blood. A tail vein injection of $10^{3}$ CFUs showed equivalent bacterial clearance in C57BL6 and gld mice (data not shown). 
Figure 1 Reduced bacterial clearance in gld mice with established pneumonia. (A) Colony-forming units (CFUs) of bacteria in lung homogenates from wild-type control mice (WT) and mice deficient in Fas ligand $(g / d)$ or Fas $(/ p r)$ $24 \mathrm{~h}$ after intratracheal instillation of $10^{7}$ CFUs of serotype 1 pneumococci; (B) CFUs of bacteria in blood in the same experiments as $(A)$; $(C)$ percentage of neutrophils (PMN) in bronchial alveolar lavage (BAL) from the same experiments as (A); (D) CFUs of bacteria in lung homogenates from mice $24 \mathrm{~h}$ after intratracheal instillation of $10^{7}$ CFUs of serotype 2 pneumococci; (E) CFUs of bacteria in blood in the same experiments as (D); (F) percentage of neutrophils in BAL from the same experiments as (D); (G) CFUs of bacteria in lung homogenates from mice $24 \mathrm{~h}$ after intratracheal instillation of $10^{7}$ CFUs of serotype 4 pneumococci; $(\mathrm{H})$ CFUs of bacteria in blood in the same experiments as (G); (I) percentage of neutrophils in $B A L$ from the same experiments as $(G)$. ( $A$, $B, D, E, G, H)$ Line at median, Kruskal-Wallis test with Dunn's multiple comparison test; $(C, F, I)$ : mean + SEM, analysis of variance with Bonferroni's multiple comparison test, ${ }^{*} \mathrm{p}<0.05$, ${ }^{* *} \mathrm{p}<0.01,{ }^{* * *} \mathrm{p}<0.001$, $\mathrm{n}=12-15$.
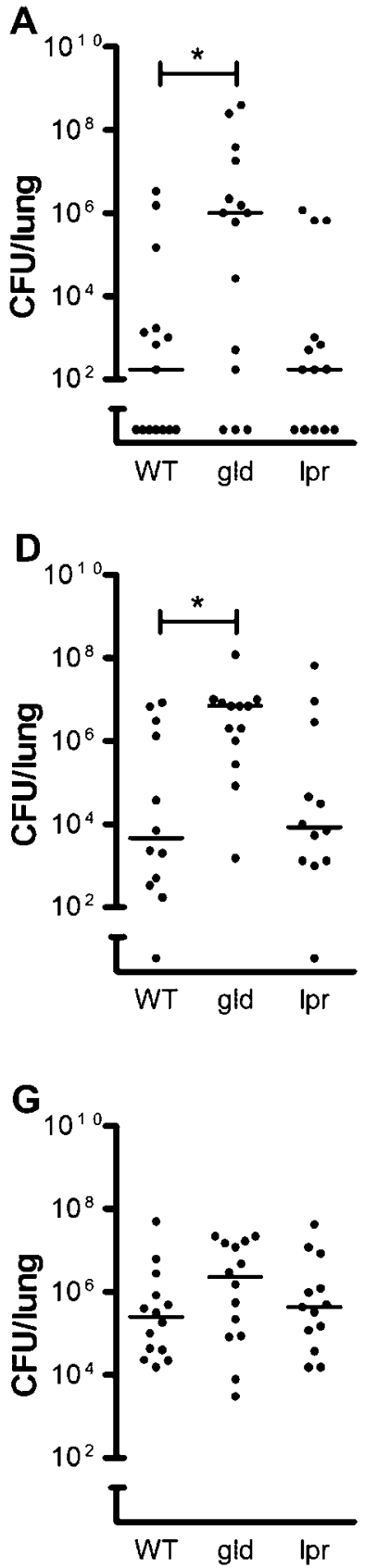
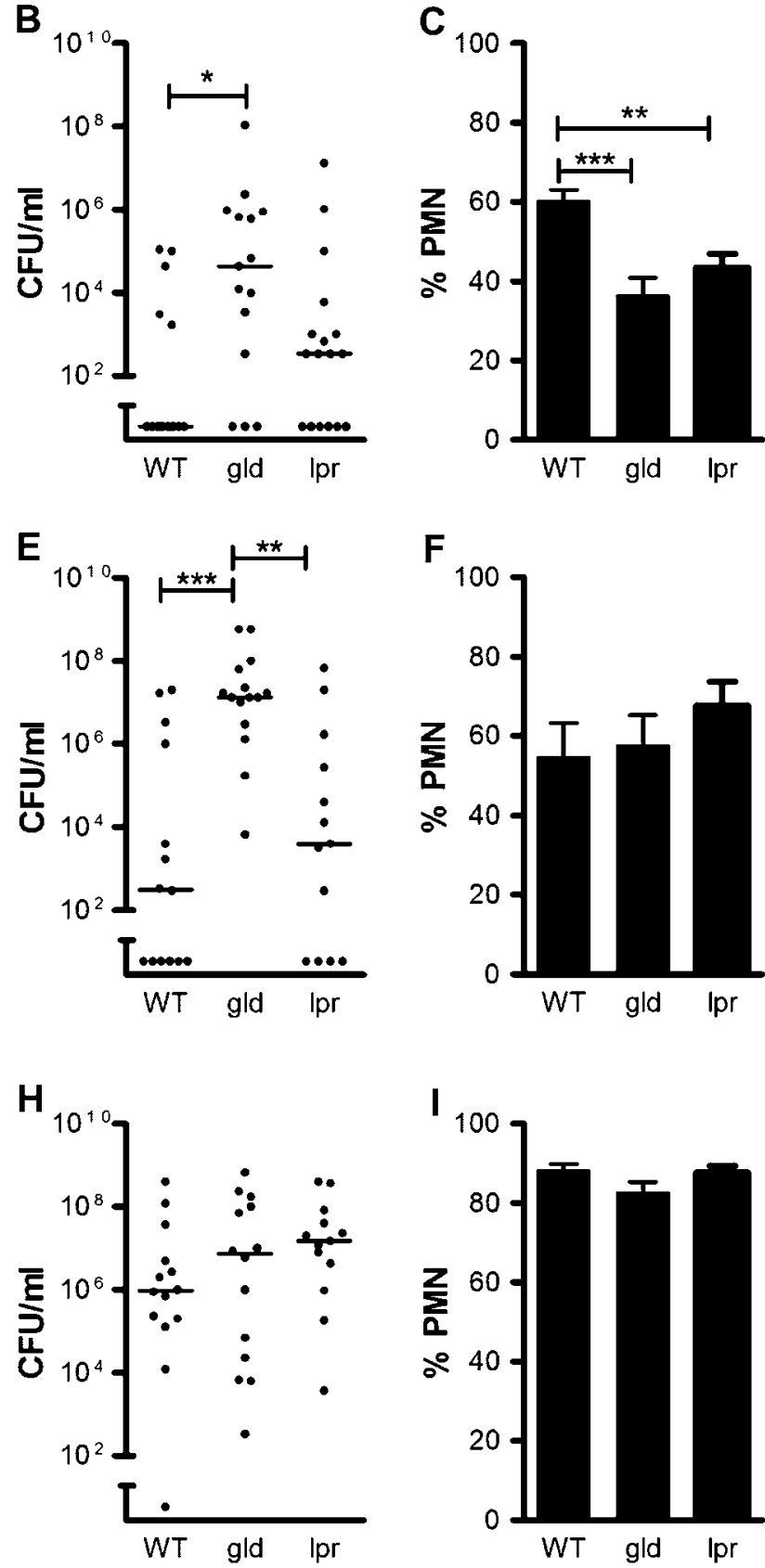

Results at $48 \mathrm{~h}$ after high-dose serotype 2 infection also revealed increased CFUs in gld mice (figure $5 \mathrm{~A}, \mathrm{~B}$ ) though to a lesser extent than at $24 \mathrm{~h}$ (figure 1D,E), with reduced neutrophil counts (figure 5C). In keeping with these findings gld mice had increased mortality in comparison to wild-type mice in the period from 72 to $120 \mathrm{~h}$ (figure $5 \mathrm{D}$ ). At $72 \mathrm{~h}$ there was $40 \%$ survival in the gld mice versus $70 \%$ in the wild-type mice and the median survival in gld mice was $72 \mathrm{~h}$ versus undefined in the wild-type mice, although overall survival did not differ $(p=0.5033)$.

\section{Alterations in the levels of T-cell activation and apoptosis in gld mice}

Since accumulation of activated $\mathrm{T}$ cells is an early feature of pneumococcal pneumonia and $\mathrm{T}$-cell responses are required for optimal clearance of bacteria, ${ }^{22} 23$ we next examined levels of Tcell activation in each mouse strain by studying levels of CD25
$\mathrm{CD}^{+} \mathrm{T}$ cells in cells isolated $24 \mathrm{~h}$ after infection. We used highdose type $4 S$ pneumoniae to ensure all mice had equivalent levels of bacteraemia. As shown there were greater levels of $\mathrm{CD}^{+} \mathrm{T}$ cell activation in gld mice (figure 6A) and these mice had lower levels of apoptotic T cells (figure 6B).

DcR3-a corrects the impairment in bacterial clearance associated with FasL deficiency

The decoy receptor DcR3 not only neutralises FasL but also additional TNFSF members LIGHT and TL1A and can modify $\mathrm{T}$ cell activation. ${ }^{24}{ }^{25}$ We reasoned if the impaired clearance of bacteria was solely due to FasL deficiency then DcR3 would replicate the gld phenotype in C57BL/6 and lpr mice but have no effect upon gld mice. To test this we utilised a modified analogue of DcR3, DcR3-a (FLINT), which is more resistant to proteolytic cleavage in vivo than the parental structure. ${ }^{6}{ }^{26}$ DcR3-a inhibits 

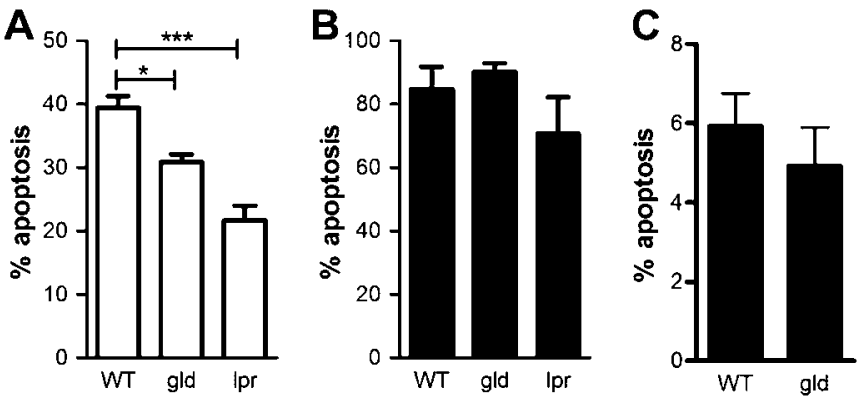

Figure 2 Mice lacking Fas ligand have reduced constitutive neutrophil apoptosis but no defect in apoptosis in the presence of bacteria. Peripheral blood neutrophils from wild-type control mice (WT) and mice deficient in Fas ligand $(g / d)$ or Fas $(/ p r)$ were isolated by negative magnetic selection. (A) Rates of apoptosis were assessed by morphology on cytospins prepared after $9 \mathrm{~h}$ of culture, $n=4$. (B) Neutrophils were infected with pneumococci $(\mathrm{MOI}=10)$ and apoptosis assessed as in $A, n=3$. (C) Apoptosis of neutrophils in bronchial alveolar lavage from WT control mice or mice deficient in Fas ligand $(g / d) 24 \mathrm{~h}$ after intratracheal instillation of $10^{7}$ colony-forming units (CFUs) of serotype 2 pneumococci, $\mathrm{n}=14 \mathrm{WT}$ and $10 \mathrm{~g} / \mathrm{d}$. Mean+SEM, analysis of variance with Bonferroni's multiple comparison test, ${ }^{*} p<0.05$, ${ }^{* * *} \mathrm{p}<0.001$.

FasL, LIGHT and TL1A in vivo and has modified bacterial clearance in other models of pneumococcal pneumonia. ${ }^{6} 26$ Surprisingly we did not find any difference in bacterial CFUs in the lungs or blood of C57BL/6 and lpr mice in the presence or absence of DcR3-a (figure 7A,B) following infection with a moderate dose of serotype 4 pneumococci, which resulted in only low levels of neutrophil recruitment (figure 7C). In contrast, the defect in bacterial clearance was corrected in the gld mice. DcR3-a also reversed the increased levels of T-cell activation observed in gld mice (figure 8).

\section{DISCUSSION}

We demonstrate that gld mice are impaired in their ability to clear bacteria from the lung in a model of pneumococcal pneumonia. We show enhanced T-cell activation is associated with localised pulmonary defects in neutrophil function in gld mice. DcR3-a, which neutralises FasL, had no effect on bacterial
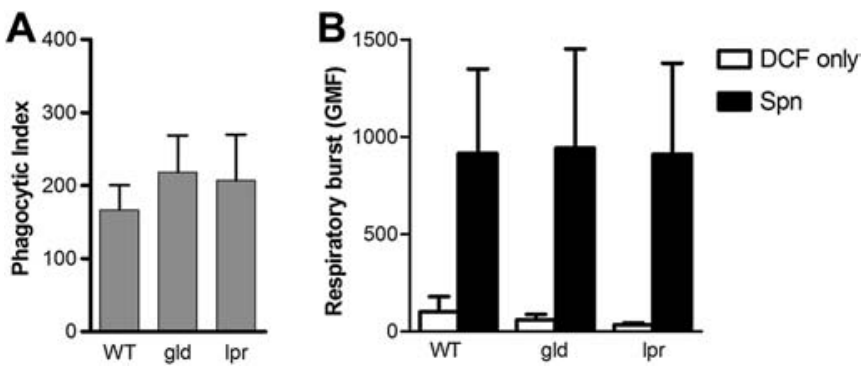

Figure 3 Mice lacking Fas ligand have preserved neutrophil function ex vitro. Peripheral blood neutrophils from wild-type control mice (WT) and mice deficient in Fas ligand $(g / d)$ or Fas $(/ p r)$ were isolated by negative magnetic selection. (A) Phagocytosis was assessed by calculating phagocytic index on cytospins of peripheral blood neutrophils incubated with opsonized zymosan $(20 \mu \mathrm{g} / \mathrm{ml})$ for $30 \mathrm{~min}, \mathrm{n}=5$. (B) Respiratory burst was assessed by flow cytometry analysis of dichlorodihydrofluorescein (DCF) fluorescence following $30 \mathrm{~min}$ of stimulation by heatkilled serotype 2 pneumococci $(\mathrm{MOI}=10)$. Geometric mean fluorescence (GMF), $n=3$. Mean+SEM, analysis of variance with Bonferroni's multiple comparison test, $\mathrm{p}=$ not significant.
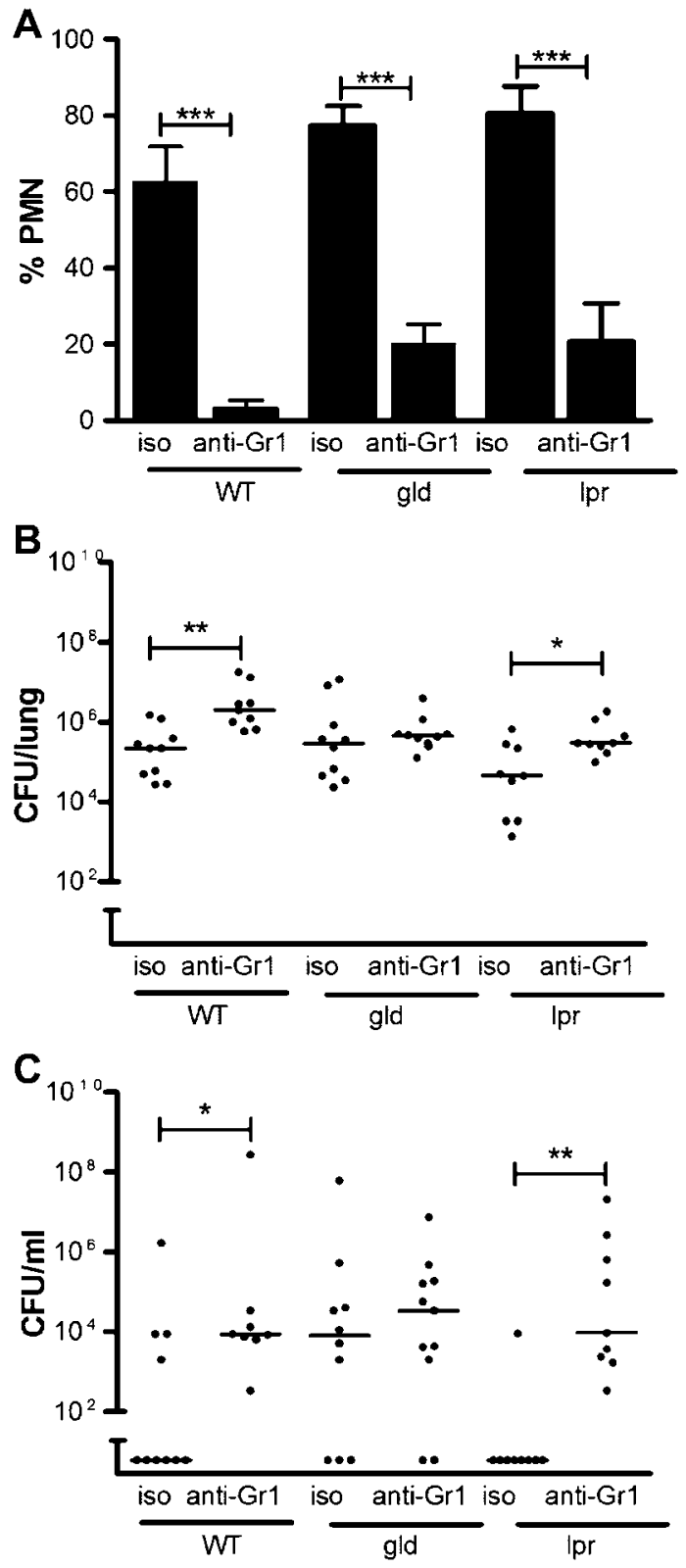

Figure 4 Absence of Fas ligand impairs neutrophil function in vivo. Wild-type control mice (WT) and mice deficient in Fas ligand $(g / d)$ or Fas (Ipr) were treated with anti-Gr1 antibody to deplete neutrophils (PMN) (anti-Gr1) or isotype control (iso) $24 \mathrm{~h}$ prior to intratracheal instillation of $10^{7}$ colony-forming units (CFUs) of serotype 2 pneumococci. Fourteen hours post infection $(A)$ the percentage of neutrophils in bronchial alveolar lavage was assessed, mean+SEM, analysis of variance with Bonferroni's multiple comparison test; (B) CFUs of bacteria in lung homogenates; (C) CFUs of bacteria in blood, line at median, Kruskal-Wallis test with Dunn's multiple comparison test, ${ }^{*} \mathrm{p}<0.05$, ${ }^{* *} \mathrm{p}<0.01$, ${ }^{* *} \mathrm{p}<0.001, \mathrm{n}=9-10$.

clearance in wild-type or $\mathrm{lpr}$ mice but corrected the defects in bacterial clearance in gld mice and reduced levels of T-cell activation, suggesting unopposed $\mathrm{T}$-cell activation by other TNF superfamily members, inhibitable by DcR3-a, influenced bacterial clearance in gld mice. These results suggest that altered balance of TNFSF signalling, involving FasL and other DcR3 ligands, adversely influences the outcome of pneumonia during a defined period when initial neutrophil and T-cell recruitment are required to control infection and at a defined threshold of infection when resident host responses fail. ${ }^{22} 23$ 
Figure 5 The difference in bacterial outcome between wild-type (WT) mice and mice deficient in Fas ligand $(g / d)$ is reduced at later time points. (A) Colonyforming units (CFUs) of bacteria in lung homogenates from WT control mice $(n=14)$ and gld mice $(n=12) 48 \mathrm{~h}$ after intratracheal instillation of $1 \times 10^{7}$ CFUs of serotype 2 pneumococci. (B) CFUs of bacteria in blood in the same experiments as $(A)$, line at median, Mann-Whitney. (C) The percentage of neutrophils (PMN) in bronchial alveolar lavage in the same experiments as (A), mean+SEM, t test, ${ }^{* *} \mathrm{p}<0.01$. (D) WT $(n=11)$ and gld mice $(n=12)$ were instilled with $10^{7}$ CFUs of type 2 pneumococci and followed for 10 days. Median survival: WT, undefined; gld, $72 \mathrm{~h}$; log-rank test $\mathrm{p}=0.5033$.
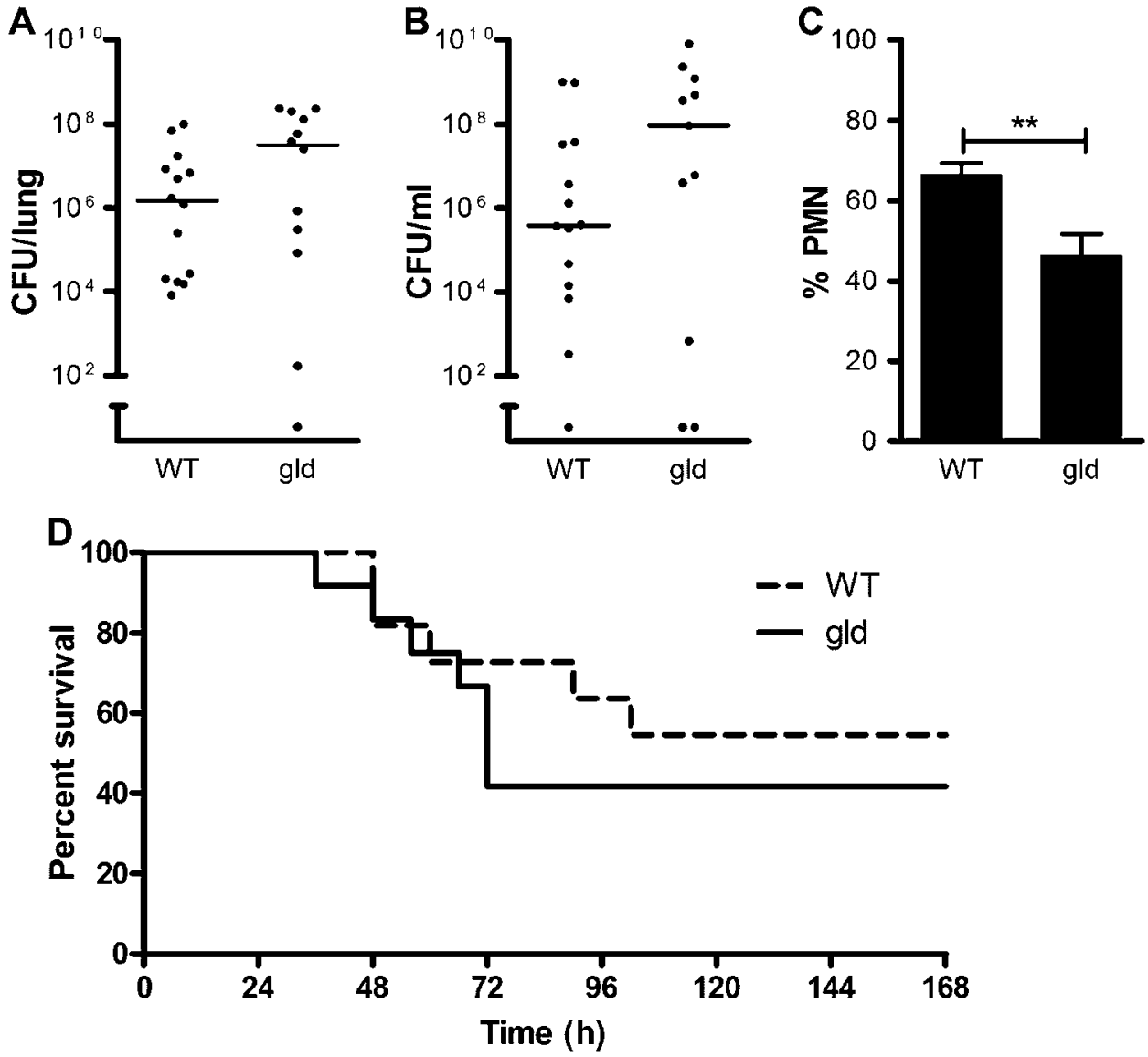

The effects of complete and selective inhibition of Fas signalling on pneumococcal pneumonia, to our knowledge, have not previously been addressed. We did not predict a difference between gld and $l p r$ mice. Both mutations are loss of function
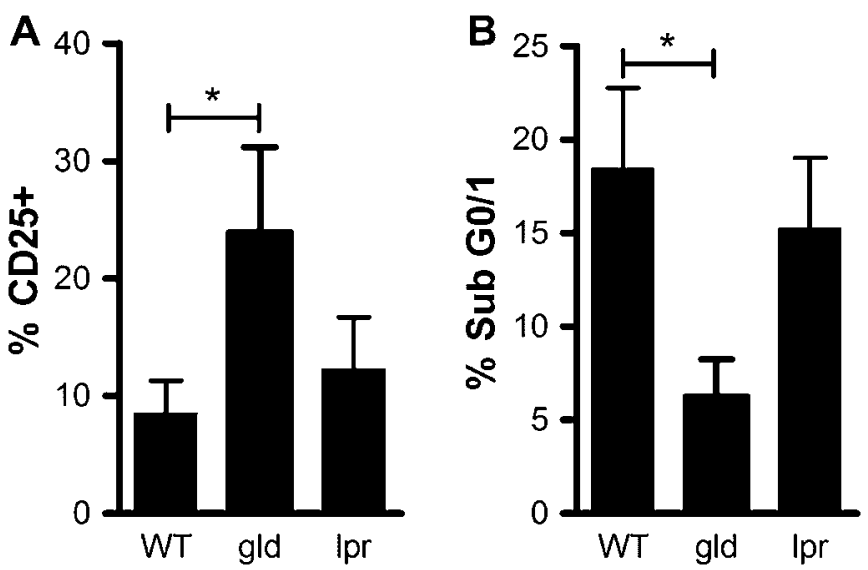

Figure 6 Alterations in levels of T-cell activation and apoptosis in mice deficient in Fas ligand $(g / d)$. Splenocytes were isolated from spleens of wild-type control mice (WT) and gld mice or mice deficient in Fas (Ipr) $24 \mathrm{~h}$ after intratracheal instillation of $10^{7}$ colony-forming units of serotype 4 pneumococci and $\mathrm{CD}^{+} \mathrm{T}$ cells identified by flow cytometry. (A) Activation was measured by the levels of CD25 cells $(n=3)$. (B) Apoptosis was measured by analysing the percentage of cells with hypodiploid DNA after propidium iodide staining (\% Sub G0/1, $n=5$ ), mean + SEM, analysis of variance with Bonferroni's multiple comparison test, ${ }^{*} \mathrm{p}<0.05$. mutations that impair Fas signalling leading to autoimmune manifestations $^{13}$ and we confirmed the development of lymphadenopathy and splenomegaly as gld and lpr mice aged. The extent of inhibition of Fas signalling in $\mathrm{lpr}$ mice is less complete than that observed in gld mice. ${ }^{12} 13$ The lpr mutation results from the insertion of an early transposable element into intron 2 and is leaky with residual low-level expression of Fas. ${ }^{12}$ In contrast, the gld mutation introduces a point mutation into the C-terminus of FasL and blocks binding of FasL to Fas. ${ }^{10}$ These result in subtle differences in the inflammatory response between strains. ${ }^{20}$ Of immediate relevance gld mice have a more profound reduction in peripheral blood $\mathrm{CD} 4^{+}, \mathrm{CD} 4^{+} \mathrm{CD} 25^{+}$ $\mathrm{FoxP}^{+}$, and most markedly $\mathrm{CD}^{+}{ }^{+} \mathrm{T} \mathrm{cells.}^{27}$ We found reduced neutrophil-dependent bacterial clearance and enhanced T-cell activation occurred only in gld mice, suggesting residual Fas signalling activity in $1 p r$ mice may have been sufficient to ensure optimal host responses and to prevent the more marked perturbations in T-cell subsets previously described in gld mice. ${ }^{27}$

We show an early requirement for FasL at the stage of pneumonia when neutrophils start to be recruited to the lung. Under these circumstances absolute and selective deficiency of FasL was associated with enhanced activation of $T$ cells and reduced pulmonary neutrophil function. Residual Fas signalling, as evidenced by $1 p r$ mice, is sufficient for host defence but complete deficiency exposes the host to harmful effects of other TNFSF members. The concept that the unopposed actions of other TNFSF members may mediate specific phenotypes during impairment of Fas signalling is not without precedent since neutralisation of TNF- $\alpha$ attenuates the generalised lymphadenopathy of gld mice. ${ }^{28}$ 

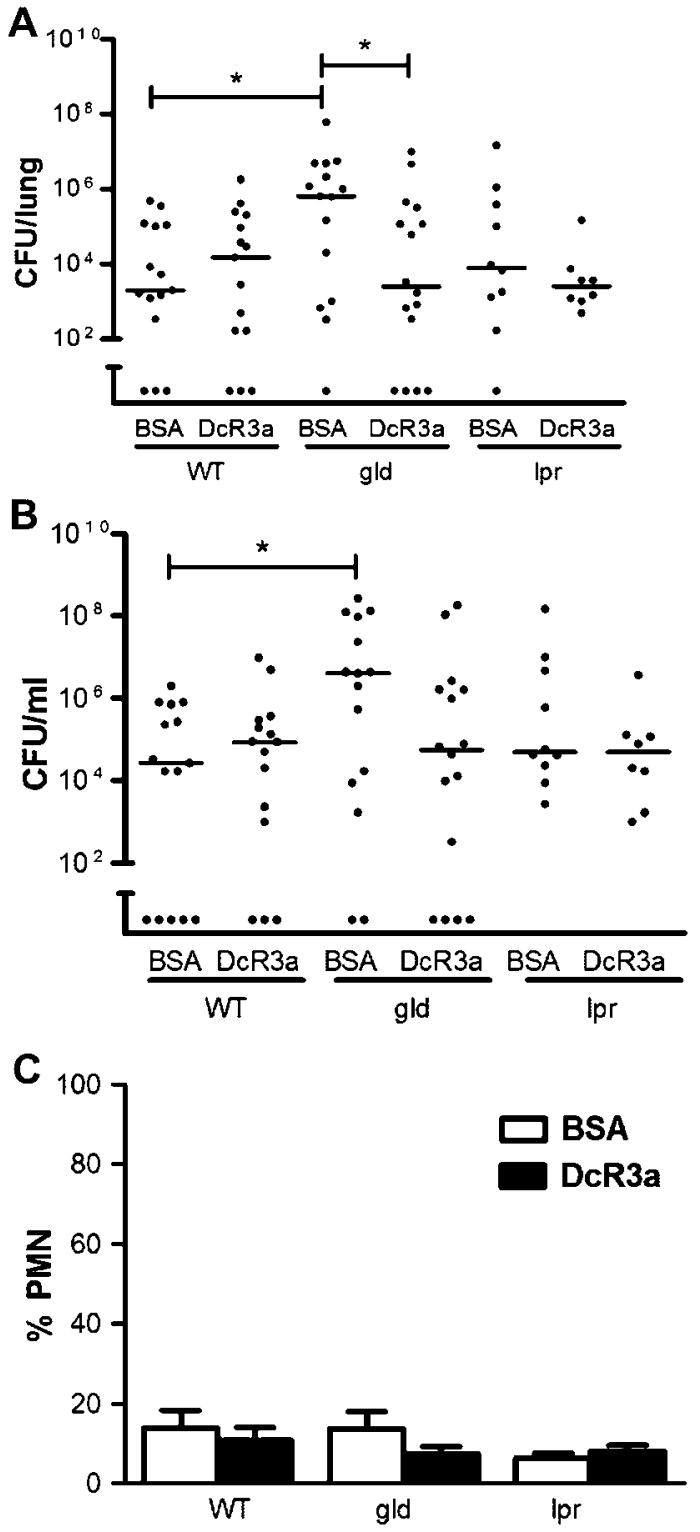

Figure 7 Decoy receptor 3 analogue (DcR3a) corrects the impairment in bacterial clearance associated with Fas ligand deficiency. Wild-type control mice (WT) and mice deficient in Fas ligand (gld) or Fas (Ipr) were treated with DcR3a or BSA control (BSA) immediately prior to intratracheal instillation of $5 \times 10^{5}$ colony-forming units (CFUs) of serotype 4 pneumococci and $12 \mathrm{~h}$ post infection. Twenty-four hours post infection, (A) CFUs of bacteria in lung homogenates. (B) CFUs of bacteria in blood in the same experiments as (A), line at median, Kruskal-Wallis test with Dunn's multiple comparison test. (C) The percentage of neutrophils (PMN) in bronchial alveolar lavage in the same experiments as $(A)$ and $(B)$ were estimated, mean + SEM, analysis of variance with Bonferroni's multiple comparison test, ${ }^{*} \mathrm{p}<0.05, \mathrm{n}=8-16$.

Fas signalling influenced neutrophil recruitment and rates of constitutive apoptosis, as previously documented. ${ }^{6}$ It did not alter rates of neutrophil apoptosis after bacterial infection. Overall this resulted in variable differences in neutrophil numbers in the lung. Differences in neutrophil numbers alone do not explain the selective impairment in host defence of the gld mice and there was no intrinsic deficit in neutrophil function in either $l p r$ or gld mice. The functional neutrophil impairment in bacterial clearance was confined to the lung since clearance from the blood was normal following intravenous challenge. We

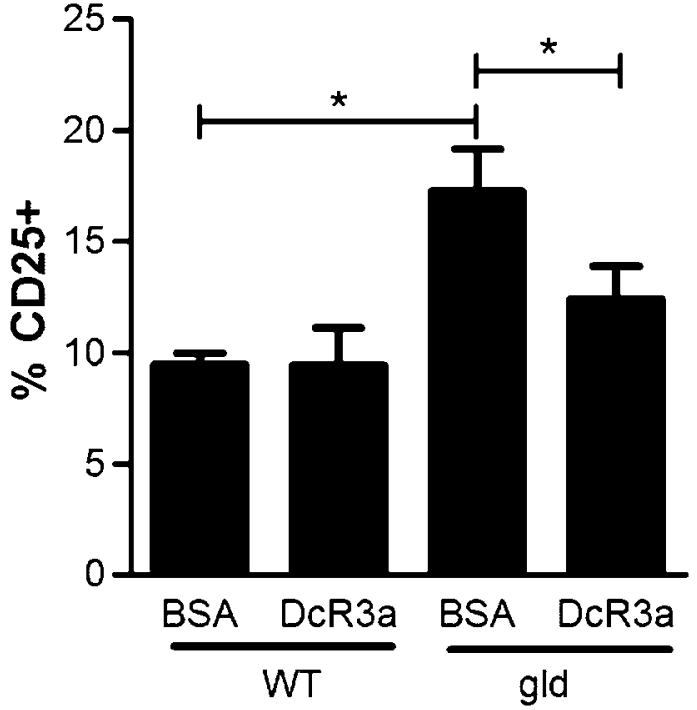

Figure 8 T-cell activation is reduced in mice deficient in Fas ligand (g/d) after decoy receptor 3 analogue (DcR3a) treatment. Wild-type control mice (WT) and gld mice were treated with DcR3a or BSA control (BSA) immediately prior to intratracheal instillation of $1 \times 10^{7}$ colonyforming units (CFUs) of serotype 4 pneumococci. Splenocytes were isolated from spleens $24 \mathrm{~h}$ after infection, and the percentage of CD25 ${ }^{+}$ $\mathrm{CD}^{+} \mathrm{T}$ cells recorded by flow cytometry, mean +SEM, analysis of variance with Bonferroni's multiple comparison test, ${ }^{*} p<0.05, n=3-6$.

conclude that there was a localised defect in the phagocytosis or killing of bacteria by pulmonary neutrophils in gld mice. Fas signalling can modify toll-like receptor responses ${ }^{27}$ but the absence of macrophage or intrinsic neutrophil defects argues against this being an important factor. The unique defects we identified in gld mice were characterised by selective alteration in pulmonary neutrophil function and were associated with increased T-cell activation.

The role of $\mathrm{T}$ cells in the innate response to pneumococcal pneumonia has been controversial. $\mathrm{T}$ helper (Th) $17 \mathrm{~T}$ cells contribute to phagocytic function during upper airway colonisation while $\mathrm{CD} 4^{+} \mathrm{T}$ cells are necessary for optimal clearance of bacteria from the lung in the initial stages of pneumonia. ${ }^{22} 23$ In contrast, selective $\mathrm{CD} 4^{+} \mathrm{T}$-cell depletion or non-selective T-cell inhibition reduces invasive disease ${ }^{29}$ but $\mathrm{CD}^{+} \mathrm{T}$-cell depletion worsens pneumonia outcome. ${ }^{30}$ Our results suggest that FasLdependent regulation of activated T cells could be a key factor in ensuring optimal pneumococcal clearance by pulmonary neutrophils early in the course of pneumonia and if this fails there is a more rapid progression to mortality. Since gld mice have relatively lower numbers of $\mathrm{CD}^{+} \mathrm{T} \mathrm{cells}^{27}$ and these cells can prominently express FasL, our results are consistent with an early role for these cells in pneumonia. Important roles for other T-cell populations such as $\mathrm{CD}^{+}{ }^{+} \mathrm{T}$-cells are equally possible in keeping with observations that $\mathrm{T}$-cell depletion in fulminant disease is beneficial, since the concept of improving outcomes by reducing host responses in more fulminant disease are well established. ${ }^{21}$ However, alterations in T-cell activation may be crucial. The gld phenotype is associated with a large population of preactivated T cells, which leads to hypersecretion of Th1 cytokines and localised perturbation of cytokine networks could alter neutrophil function. ${ }^{31}$ Excess T-cell activation harms host defence; Th1-derived cytokines impair innate function when present at inappropriately high or sustained levels; interferon $\gamma$ can inhibit Fc $\gamma$-mediated phagocytosis and intracellular killing of bacteria. ${ }^{32} 33$ Therefore excessive immune activation may 
have a paradoxical effect on innate responses to $S$ pneumoniae in the lung and be as harmful as deficiency. Reducing T-cell activation during selective FasL deficiency or in fulminant disease can improve pneumonia outcome. ${ }^{29}$

Termination of immune responses requires FasL-induced T-cell apoptosis during sustained responses to micro-organisms. ${ }^{8}$ DcR3-a reduced T-cell activation and the defect in neutrophildependent bacterial clearance in gld mice, suggesting unopposed LIGHT/TL1A mediate the inappropriate T-cell activation in gld mice. Consistent with this possibility T-cell derived LIGHT/TL1A drive Th1 and Th17 cytokine expression at mucosal surfaces ${ }^{34} 35$ and favour dysregulated inflammatory responses. ${ }^{36} 37$ Although DcR3-a could alter neutrophil or macrophage responses directly the absence of evidence of macrophage or intrinsic neutrophil defects make this unlikely.

The benefits of FasL in our model are consistent with requirements for FasL to regulate $\mathrm{T}$-cell activation during the early evolution of pulmonary neutrophil responses. The results of Matute-Bello et al suggest incomplete (Ipr mice) or nonselective inhibition (DcR3-a) of Fas at a later stage of pneumococcal pneumonia improve outcomes. ${ }^{6}$ Reduction of neutrophil numbers or function, when these exceed the optimal amount required for bacterial clearance, can improve outcome $e^{2138}$ and we believe the effects Matute-Bello et al observed are consistent with this requirement to decrease neutrophil responses. We found that Ipr or DcR3-a-treated wild-type mice had no worse outcome than wild-type mice, while only the complete but selective inhibition of FasL in gld mice worsened outcome in all but the most advanced stages of pneumonia. It therefore seems that FasL signalling and that of related TNFSF ligands must be finely tuned to ensure optimal host responses during pneumonia (online supplementary figure 4).

Examination of clinical cohorts will be required to tease out these paradigms and confirm the therapeutic potential in CAP. Although lpr/gld mice are useful models of immune homeostasis and clinical diseases, as the murine phenotype varies considerably with genetic background, translation in clinical studies is essential. ${ }^{8}$ The potential translational implications are that manipulation of TNFSF ligands, an emerging therapeutic approach in oncology and other settings, ${ }^{39}$ could improve the outcome of CAP. Increased FasL expression has been documented in patients with pneumococcal infection. ${ }^{5}{ }^{6}$ Documentation of relative decreases in FasL expression, altered ratios of DcR3 ligand expression leading to increased LIGHT/TL1A relative to FasL or enhanced T-cell activation in patients with worse clinical outcomes could trigger TNFSF ligand neutralisation using DcR-3 neutralisation or similar approaches early in pneumonia. This approach might also be beneficial when there is excessive neutrophilic inflammation later in disease. Our findings suggest that $\mathrm{T}$-cell activation must be tightly controlled and confirm an important role for FasL expression in regulating host responses during pneumococcal pneumonia. These results also suggest modulation of DcR3 ligands could represent an unexploited therapeutic strategy that improves outcomes during CAP.

Contributors Conception hypothesis and design: HMM, PGH, MKW and DHD; data acquisition and analysis: HMM, MD, AART, SRW, SG, DRW, VJW, MKW and DHD; manuscript preparation: HMM, MKW and DHD.

Funding This work was supported by a grant from the Sheffield Hospitals Charitable Trust Research Grant (No. 7843), a Wellcome Trust Senior Clinical Fellowship to DHD (No. 076945) and a British Lung Foundation fellowship to HMM (F05/7). SRW is a Wellcome Clinician Scientist (No. 078244) and AART an MRC Clinical Research Training Fellow (G0802255).

Competing interests DRW and VJW are employees and have stock in Eli Lilly \& Company.

Provenance and peer review Not commissioned; externally peer reviewed.

\section{REFERENCES}

1. O'Brien KL, Wolfson LJ, Watt JP, et al. Burden of disease caused by Streptococcus pneumoniae in children younger than 5 years: global estimates. Lancet 2009;374:893-902.

2. Nagata S. Fas ligand-induced apoptosis. Annu Rev Genet 1999;33:29-55

3. Liles WC, Kiener PA, Ledbetter JA, et al. Differential expression of Fas (CD95) and Fas ligand on normal human phagocytes: implications for the regulation of apoptosis in neutrophils. J Exp Med 1996;184:429-40.

4. Matute-Bello G, Liles WC, Steinberg KP, et al. Soluble Fas ligand induces epithelial cell apoptosis in humans with acute lung injury (ARDS). J Immunol 1999:163:2217-25.

5. Kemp K, Bruunsgaard H, Skinhoj P, et al. Pneumococcal infections in humans are associated with increased apoptosis and trafficking of type 1 cytokine-producing $T$ cells. Infect Immun 2002;70:5019-25.

6. Matute-Bello G, Liles WC, Frevert CW, et al. Blockade of the Fas/FasL system improves pneumococcal clearance from the lungs without preventing dissemination of bacteria to the spleen. J Infect Dis 2005;191:596-606.

7. Matute-Bello G, Frevert CW, Liles WC, et al. Fas/Fas ligand system mediates epithelial injury, but not pulmonary host defenses, in response to inhaled bacteria. Infect Immun 2001;69:5768-76.

8. Strasser A, Jost PJ, Nagata $\mathrm{S}$. The many roles of FAS receptor signaling in the immune system. Immunity 2009:30:180-92.

9. Dockrell DH. Apoptotic cell death in the pathogenesis of infectious diseases. $J$ Infect 2001:42:227-34.

10. Takahashi T, Tanaka M, Brannan $\mathrm{Cl}$, et al. Generalized lymphoproliferative disease in mice, caused by a point mutation in the Fas ligand. Cell 1994;76:969-76.

11. Watanabe-Fukunaga R, Brannan $\mathrm{Cl}$, Copeland NG, et al. Lymphoproliferation disorder in mice explained by defects in Fas antigen that mediates apoptosis. Nature 1992;356:314-17.

12. Kobayashi S, Hirano T, Kakinuma M, et al. Transcriptional repression and differentia splicing of Fas mRNA by early transposon (ETn) insertion in autoimmune lpr mice. Biochem Biophys Res Commun 1993;191:617-24.

13. Nagata S, Suda T. Fas and Fas ligand: Ipr and gld mutations. Immunol Today 1995;16:39-43.

14. Nijhawan D, Fang M, Traer $\mathrm{E}$, et al. Elimination of $\mathrm{Mcl}-1$ is required for the initiation of apoptosis following ultraviolet irradiation. Genes Dev 2003:17:1475-86.

15. Yu KY, Kwon B, Ni J, et al. A newly identified member of tumor necrosis factor receptor superfamily (TR6) suppresses LIGHT-mediated apoptosis. J Biol Chem 1999:274:13733-6.

16. Migone TS, Zhang J, Luo X, et al. TL1A is a TNF-like ligand for DR3 and TR6/DcR3 and functions as a T cell costimulator. Immunity 2002;16:479-92.

17. Dockrell DH, Marriott HM, Prince LR, et al. Alveolar macrophage apoptosis contributes to pneumococcal clearance in a resolving model of pulmonary infection. $\mathrm{J}$ Immunol 2003:171:5380-8.

18. Walmsley SR, Chilvers ER, Thompson AA, et al. Prolyl hydroxylase 3 (PHD3) is essential for hypoxic regulation of neutrophilic inflammation in humans and mice. J Clin Invest 2011;121:1053-63.

19. Nicoletti I, Migliorati G, Pagliacci MC, et al. A rapid and simple method for measuring thymocyte apoptosis by propidium iodide staining and flow cytometry $\mathrm{J}$ Immunol Methods 1991:139:271-9.

20. Neff TA, Guo RF, Neff SB, et al. Relationship of acute lung inflammatory injury to Fas/FasL system. Am J Pathol 2005;166:685-94.

21. Marks M, Burns T, Abadi $\mathbf{M}$, et al. Influence of neutropenia on the course of serotype 8 pneumococcal pneumonia in mice. Infect Immun 2007;75:1586-97.

22. Zhang Z, Clarke TB, Weiser JN. Cellular effectors mediating Th17-dependent clearance of pneumococcal colonization in mice. J Clin Invest 2009;119:1899-909.

23. Kadioglu A, Coward W, Colston MJ, et al. CD4-T-lymphocyte interactions with pneumolysin and pneumococci suggest a crucial protective role in the host response to pneumococcal infection. Infect Immun 2004;72:2689-97.

24. Han B, Wu J. DcR3 protects islet beta cells from apoptosis through modulating Adcyap1 and Bank1 expression. J Immunol 2009;183:8157-66.

25. Hsu TL, Wu YY, Chang YC, et al. Attenuation of Th1 response in decoy receptor 3 transgenic mice. J Immunol 2005:175:5135-45.

26. Wroblewski VJ, Witcher DR, Becker GW, et al. Decoy receptor 3 (DcR3) is proteolytically processed to a metabolic fragment having differential activities agains Fas ligand and LIGHT. Biochem Pharmacol 2003:65:657-67.

27. Omokaro So, Desierto MJ, Eckhaus MA, et al. Lymphocytes with aberrant expression of Fas or Fas ligand attenuate immune bone marrow failure in a mouse model. J Immunol 2009;182:3414-22.

28. Korner H, Cretney E, Wilhelm P, et al. Tumor necrosis factor sustains the generalized lymphoproliferative disorder (gld) phenotype. J Exp Med 2000;191:89-96.

29. LeMessurier $\mathbf{K}$, Hacker $\mathrm{H}$, Tuomanen $\mathrm{E}$, et al. Inhibition of $\mathrm{T}$ cells provides protection against early invasive pneumococcal disease. Infect Immun 2010;78:5287-94.

30. Weber SE, Tian H, Pirofski LA. CD8 + cells enhance resistance to pulmonary serotype 3 Streptococcus pneumoniae infection in mice. J Immunol 2011;186:432-42.

31. Davidson WF, Calkins C, Hugins A, et al. Cytokine secretion by C3H-lpr and -gld T cells. Hypersecretion of IFN-gamma and tumor necrosis factor-alpha by stimulated CD4+ T cells. J Immunol 1991;146:4138-48.

32. Speert DP, Thorson L. Suppression by human recombinant gamma interferon of in vitro macrophage nonopsonic and opsonic phagocytosis and killing. Infect Immun 1991;59:1893-8

33. Sun K, Metzger DW. Inhibition of pulmonary antibacterial defense by interferongamma during recovery from influenza infection. Nat Med 2008;14:558-64. 
34. Cohavy 0, Zhou J, Granger SW, et al. LIGHT expression by mucosal T cells may regulate IFN-gamma expression in the intestine. J Immunol 2004;173:251-8.

35. Papadakis KA, Zhu D, Prehn JL, et al. Dominant role for TL1A/DR3 pathway in IL-12 plus IL-18-induced IFN-gamma production by peripheral blood and mucosal CCR9+ T lymphocytes. J Immunol 2005;174:4985-90.

36. Jungbeck M, Daller B, Federhofer J, et al. Neutralization of LIGHT ameliorates acute dextran sodium sulphate-induced intestinal inflammation. Immunology 2009;128:451-8.
37. Shih DQ, Barrett R, Zhang X, et al. Constitutive TL1A (TNFSF15) expression on lymphoid or myeloid cells leads to mild intestinal inflammation and fibrosis. PloS One 2011;6:e16090.

38. Marriott HM, Jackson LE, Wilkinson TS, et al. Reactive oxygen species regulate neutrophil recruitment and survival in pneumococcal pneumonia. Am J Respir Crit Care Med 2008:177:887-95.

39. Johnstone RW, Frew AJ, Smyth MJ. The TRAIL apoptotic pathway in cancer onset, progression and therapy. Nat Rev Cancer 2008;8:782-98. 